\title{
Transcatheter creation and enlargement of ventricular septal defects for relief of ventricular hypertension
}

\author{
Jeffery Meadows, MD, ${ }^{a}$ Frank Pigula, MD, James Lock, MD, ${ }^{\mathrm{a}, \mathrm{c}}$ and Audrey Marshall, MD ${ }^{\mathrm{a}, \mathrm{c}}$
}

Objective: Creation or enlargement of a ventricular septal defect is indicated in rare clinical situations. In the setting of double-outlet right ventricle requiring singleventricle palliation, left ventricular outflow tract obstruction caused by progressive restriction at the ventricular septal defect poses an uncommon but recognized dilemma. In this situation surgical ventricular septal defect enlargement may be desirable but risks damage to the atrioventricular valve or conduction system. We report the results of a novel technique for transcatheter creation or enlargement of ventricular septal defects as an alternative to reoperation when decompression of an isolated ventricle is indicated.

Methods: Eight patients had undergone transcatheter ventricular septal defect enlargement or creation, and 3 of these patients had undergone 4 prior surgical attempts at left ventricular decompression. Ventricular aneurysms had developed in 3 patients before intervention.

Results: Five patients underwent ventricular septal defect creation, and 3 patients underwent enlargement of existing ventricular septal defects. Initial intervention resulted in a decreased ventricular septal defect pressure gradient from $76.9 \mathrm{~mm} \mathrm{Hg}$ to $20.3 \mathrm{~mm} \mathrm{Hg}(P=.004)$. There was no procedural mortality or sustained heart block. Two patients had moderate-to-severe atrioventricular valve regurgitation, and 1 required surgical repair. At last follow-up, all ventricular septal defects remained patent, with recurrent obstruction in the majority of cases caused by muscular hypertrophy beyond the stent margins. In 1 patient a ventricular aneurysm has regressed in size. Repeat intervention reduced recurrent obstruction, but recurrence was the rule.

Conclusions: When reoperation is considered high risk, transcatheter creation and enlargement of ventricular septal defects is possible with excellent short-term results. Recurrent obstruction is common but responds to repeated intervention.

From the Departments of Cardiology a and Cardiovascular Surgery, ${ }^{\mathrm{b}}$ Children's HospitalBoston, and the Department of Pediatrics, Harvard Medical School, Boston, Mass.

Audrey Marshall reports grant support from Johnson and Johnson, the manufacturer of the stents reported on in this article.

Received for publication Aug 7, 2006; revisions received Sept 15, 2006; accepted for publication Sept 28, 2006.

Address for reprints: Jeffery Meadows, MD, Department of Cardiology, Children's Hospital-Boston, 300 Longwood Ave, Boston, MA 02115 (E-mail: Jeffrey.Meadows@ cardiochboston.org).

J Thorac Cardiovasc Surg 2007;133:912-8

$0022-5223 / \$ 32.00$

Copyright (C) 2007 by The American Association for Thoracic Surgery

doi:10.1016/j.jtcvs.2006.09.037
Further studies are required to establish clinical benefit.

$\mathrm{C}$ reation or enlargement of a therapeutic ventricular septal defect (VSD) is indicated in rare clinical situations. Among these are patients with a doubleoutlet right ventricle (DORV) requiring surgical palliation to a singleventricle physiology, in whom left ventricular (LV) outflow tract obstruction caused by progressive restriction at the VSD poses an uncommon but recognized clinical scenario. In this situation the isolated left ventricle becomes hypertensive and hypertrophied and may be prone to arrhythmia or aneurysm formation. Decompression of the left ventricle might prevent or ameliorate these potential complications and improve systemic ventricular function through the relief of adverse ventricular interaction. However, although ventricular decompression might be desirable, surgical VSD enlargement risks damage to the atrioventricular valves (AVVs) or conduction system. We report a novel technique for transcatheter creation or enlargement of VSDs that presents a minimally invasive alternative to reoperation when decompression of an isolated ventricle is indicated. 


\author{
Abbreviations and Acronyms \\ $\mathrm{AVV}=$ atrioventricular valve \\ DORV $=$ double-outlet right ventricle \\ $\mathrm{LV}=$ left ventricular \\ VSD = ventricular septal defect
}

\section{Materials and Methods}

After departmental and institutional review board approval, we reviewed departmental databases for all patients having had transcatheter VSD creation or enlargement. Patients with restrictive bulboventricular foramen, systemic ventricular outflow tract obstruction, or both were excluded. The remaining 8 patients, all with DORV and cleft or straddling AVV or analogous physiology resulting in an isolated nonsystemic ventricle, comprise the group described in this report, representing a single institution's experience spanning from 1993 through 2006. Patients presented at several stages of univentricular palliation. Underlying cardiac anatomy, prior surgical interventions, and mechanism of obstruction, as assessed by means of two-dimensional echocardiography, are shown in Table 1, in which patients are arranged in chronologic order by the date of initial cardiac catheterization.

All 8 patients had hypertensive, isolated nonsystemic ventricles. Three patients had aneurysms of the isolated ventricle, and 1 patient had sustained a cerebrovascular accident as a result of thrombus formation in the aneurysm. Three patients had undergone 4 prior surgical attempts at decompression of the hypertensive ventricle, including 2 attempts at surgical VSD enlargement, attempted division of mitral valve attachments, and creation of a Damus-Kay-Stansel anastomosis.

\section{Technical Considerations}

Catheterization procedures were performed after achievement of general anesthesia in 6 patients and during conscious sedation in 2 patients. Access for intervention was from the femoral vessels. Systemic anticoagulation was administered as heparin (100 U/kg load) with repeat doses to maintain an activated clotting time of greater than 200 seconds. Access to the hypertensive ventricle was antegrade in all cases and across the fenestration in patients having undergone the Fontan procedure. Attempting to minimize the possibility of traumatic damage to the mitral valve during the intervention, the valve was initially crossed with an inflated balloon end-hole catheter. Baseline ventriculography was performed from both ventricles and in multiple planes to characterize ventricular septal anatomy. The decision to create a VSD or enlarge the existing VSD was based on anatomic consideration of the AVV apparatus and the anticipated location of the conduction system. In cases of VSD creation, midmuscular sites, remote from atrioventricular and semilunar valves, were targeted.

TABLE 1. Anatomic and clinical characteristics

\begin{tabular}{|c|c|c|c|}
\hline Patient & Anatomy & Prior operation & Mechanism of obstruction \\
\hline$A$ & $\begin{array}{l}\text { D-malposition of the great arteries, DORV, straddling } \\
\text { cleft mitral valve with left ventricular hypoplasia }\end{array}$ & $\begin{array}{l}\text { PA banding, atrial septectomy; Fontan } \\
\text { procedure with ligation of MPA }\end{array}$ & Muscular restriction \\
\hline B & $\begin{array}{l}\text { D-malposition of the great arteries, DORV, mild } \\
\text { pulmonary stenosis, interrupted aortic arch, LJAA }\end{array}$ & Interrupted aortic arch repair, PA banding & Muscular restriction \\
\hline C & $\begin{array}{l}\text { Congenitally corrected transposition, straddling } \\
\text { tricuspid valve, hypoplastic RV, coarctation }\end{array}$ & $\begin{array}{l}\text { PA banding, coarctation repair; } \\
\text { Bidirectional Glenn operation; Fontan } \\
\text { procedure; DKS* }\end{array}$ & Muscular restriction \\
\hline $\mathrm{D}$ & $\begin{array}{l}\text { D-malposition of the great arteries, DORV, } \\
\text { hypoplastic cleft mitral valve }\end{array}$ & PA banding & AVV attachments to IVS \\
\hline $\mathrm{E}$ & $\begin{array}{l}\text { D-malposition of the great arteries, membranous } \\
\text { VSD, hypoplastic cleft mitral valve and LV, LVOTO }\end{array}$ & $\begin{array}{l}\text { Right modified BTS; bidirectional Glenn } \\
\text { operation, atrial septectomy, } \\
\text { enlargement of VSD, }{ }^{*} \text { oversewing of } \\
\text { MPA; Fontan procedure with } \\
\text { enlargement of VSD* }\end{array}$ & AVV attachments to IVS \\
\hline $\mathrm{F}$ & $\begin{array}{l}\text { D-malposition of the great arteries, DORV, pulmonary } \\
\text { atresia, straddling cleft mitral valve, LSVC }\end{array}$ & $\begin{array}{l}\text { Right modified BTS; bidirectional Glenn } \\
\text { operation }\end{array}$ & AVV attachments to IVS \\
\hline G & $\begin{array}{l}\text { tAVC, mitral valve and LV hypoplasia, complex } \\
\text { LVOTO and coarctation, LSVC }\end{array}$ & $\begin{array}{l}\text { DKS, coarctation repair and arch } \\
\text { augmentation, right modified BTS; } \\
\text { bilateral directional Glenn operation, } \\
\text { Fontan procedure }\end{array}$ & AVV attachments to IVS \\
\hline $\mathrm{H}$ & $\begin{array}{l}\text { Dextrocardia, situs inversus with L-loop ventricles } \\
\text { and L-transposition of the great arteries, cleft } \\
\text { mitral valve, complex LVOTO, LV hypoplasia }\end{array}$ & $\begin{array}{l}\text { Left modified BTS, atrial septectomy, PDA } \\
\text { ligation; takedown mitral valve } \\
\text { attachments, }{ }^{*} \text { BDG }\end{array}$ & AVV attachments to IVS \\
\hline
\end{tabular}

DORV, Double-outlet right ventricle; $P A$, pulmonary artery; $M P A$, main pulmonary artery; $L J A A$, left juxtaposition of the atrial appendages; $R V$, right ventricle; $D K S$, Damus-Kay-Stansel anastomosis; $A V V$, atrioventricular valve; IVS, intraventricular septum; VSD, ventricular septal defect; $L V$, left ventricle; $L V O T O$, left ventricular outflow tract obstruction; BTS, Blalock-Taussig shunt; $L S V C$, left superior vena cava; $t A V C$, transitional atrioventricular canal. *Surgical attempts to relieve ventricular hypertension. 


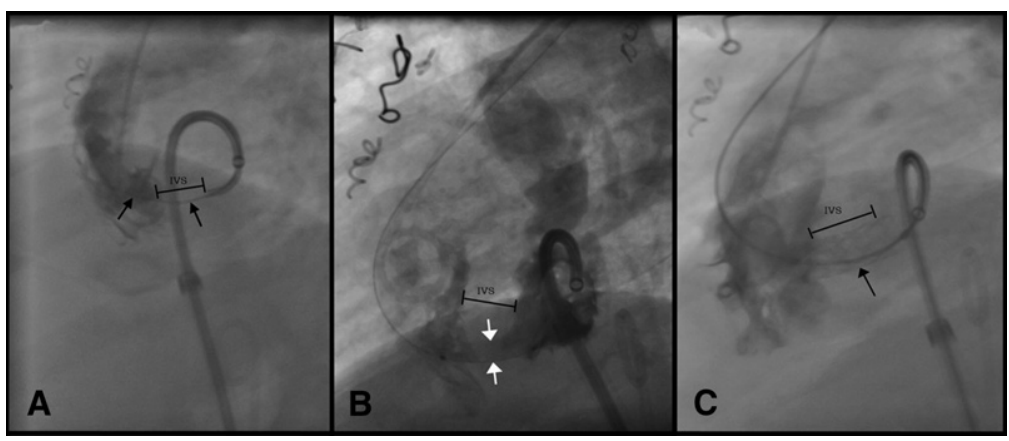

Figure 1. Transcatheter ventricular septal defect (VSD) creation and stenting: creation and stenting of a VSD in a patient with a transitional atrioventricular canal, hypoplastic mitral valve, complex left ventricular outflow tract obstruction, and a restrictive VSD caused by obstructive attachments of the mitral valve to the ventricular septum. A, Right ventricular cine angiography during perforation of the ventricular septum with the stiff end of a 0.018-inch torque wire (black arrows). The wire has been exchanged for an exchange-length floppy wire that has successfully traversed the intraventricular septum and right ventricular chamber. $B$, After balloon dilation, a newly created VSD of moderate size is demonstrated (white arrows). A hypoplastic mitral valve and complex left ventricular outflow tract obstruction are also seen. C, Stenting of the newly created VSD results in a good-size interventricular communication, decompressing the previously hypertensive left ventricle.

Once a target site was chosen, a coaxial telescoping system of catheter and long sheath with compounded curves was selected and assembled to deliver a wire to the target site. Two different wire techniques were used to tunnel through the muscular septum. The first used a 0.014-inch radiofrequency wire. The second consisted of the hand-bent stiff end of either a 0.014-inch or 0.018inch wire. Both radiofrequency wire attempts failed because of repeated loss of wattage at the tip of the wire as it was advanced through the septum in combination with the inability to control the advance of the wire tip. All successful procedures involved the serial advancement of the stiff end of a wire through a shaped woven catheter within a shaped long sheath. By using repeated angiographic reassessment and redirection, the stiff end of the wire was advanced stepwise across the ventricular septum until free within the systemic ventricle (Figure 1,A). Over this stiff portion of the wire, the tip of the woven catheter was embedded in the VSD tract, and the wire was removed and replaced with the flexible end of a new floppy wire. This exchange-length wire was passed through the created VSD, and distal position was established with the wire tip free in the aorta or looped in the systemic ventricle. Dilations beginning with 2.5-, 3-, or 4-mm balloons (Ninja, Sub-4, or Schwarten) were then used to enlarge the created VSD (Figure 1, B). Stent deployment followed in the majority of cases with either Johnson and Johnson Iliac or Genesis Premounted stents (Figure 1, C).

For enlargement of existing VSDs, the technique for long sheath placement was similar to that described above. However, in these cases the appropriate woven catheter was positioned within the hypertensive ventricle, and the VSD was crossed by using the floppy end of a 0.035 -inch torque wire. Dilation was performed with 8-, 12-, or 15-mm VACAS balloons. Stent deployment was performed with Johnson and Johnson Iliac stents.

All patients having undergone VSD stenting received periprocedural antibiotics and were maintained on heparin over the night after the procedure. Those with ventricular aneurysms were transitioned to warfarin therapy before discharge.

\section{Statistical Methods}

Statistical calculations were performed by using standard available statistical software (SPSS for Mac version 11). Comparison of means was performed by using the 2-sided paired-samples $t$ test.

\section{Results}

\section{Results of Initial Intervention}

Of the 8 patients who underwent transcatheter interventions for creation or enlargement of VSDs, 2 had repeated catheterizations for ventricular decompression. At the time of initial catheterization, patient age ranged from 9 months to 15 years (weight, 7.7-50 kg). Three patients underwent enlargement of an existing VSD, and 5 patients had creation of a new defect. One patient also underwent stenting of a muscular subaortic stenosis in an attempt to relieve LV hypertension.

Preprocedural echocardiography demonstrated qualitatively normal systolic function of the systemic ventricle in 7 of 8 patients and mild dysfunction in 1 patient. Qualitative systolic function of the hypertensive ventricle was normal in 4 patients, mildly decreased in 2 patients, and moderately to severely decreased in 2 patients. Doppler estimation of VSD maximum instantaneous pressure gradient (available in 5/8 patients) was 20 to $105 \mathrm{~mm} \mathrm{Hg}$. The size of the preexisting VSD (in 4 patients) was $3,3.5,4$, and $7 \mathrm{~mm}$, respectively, as measured by the width of the Doppler jet.

At initial catheterization, the mean systolic pressure in the isolated ventricle was $170 \mathrm{~mm} \mathrm{Hg}$ (range, 115-262 $\mathrm{mm} \mathrm{Hg}$ ), with a peak systolic gradient across the ventricular septum averaging $76.9 \mathrm{~mm} \mathrm{Hg}$ (range, 30-163 mm Hg). The cardiac index was $3.2 \mathrm{~L} \cdot \min ^{-1} \cdot \mathrm{m}^{-2}$ (range. 1.6$4.7 \mathrm{~L} \cdot \min ^{-1} \cdot \mathrm{m}^{-2}$ ). Three patients had aneurysms of the hypertensive ventricle, but there was no correlation between the presence of an aneurysm and systolic pressure in the isolated ventricle. 


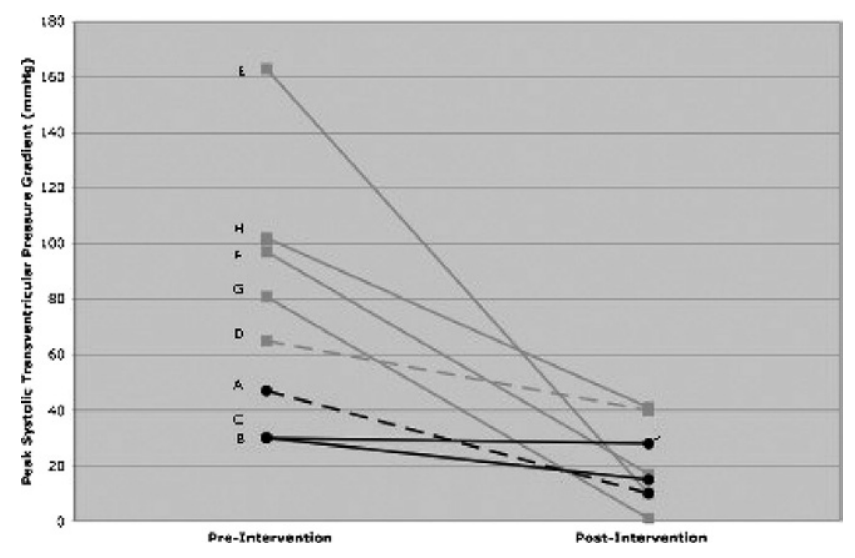

Figure 2. Results of initial intervention. Black circles indicate patients who underwent transcatheter enlargement of existing ventricular septal defects (VSDs). Gray squares indicate patients who underwent transcatheter VSD creation. Solid lines indicate procedures in which stenting occurred. Dashed lines indicate procedures involving balloon dilation without stenting. *Patient also underwent stenting of nonsystemic ventricular outflow tract obstruction.

After VSD creation or enlargement, the transventricular systolic pressure gradient decreased from $76.9 \mathrm{~mm} \mathrm{Hg}$ to $20.3 \mathrm{~mm} \mathrm{Hg}(P=.004)$, decreasing the systemic/nonsystemic ventricular pressure ratio from 1.8 to 1.2 (Figure 2). In 1 patient midcavitary obstruction of the hypertensive ventricle resulted in a residual intracavitary gradient of $87 \mathrm{~mm}$ $\mathrm{Hg}$, despite only a $10 \mathrm{~mm} \mathrm{Hg}$ gradient across the ventricular septum.

Patients in whom existing VSDs were enlarged tended to have lower degrees of ventricular hypertension compared with those in whom VSDs were created (isolated/systemic ventricle systolic pressure ratio, 1.4 vs. 2.1 ), and the magnitude of change after intervention was proportionally lower (51\% decrease vs $79 \%$ decrease in transventricular gradient).

\section{Results of Repeated Intervention}

Two patients underwent repeated interventions. One patient underwent 2 reinterventions 7 and 19 months after initial VSD creation and stenting. During the 2 additional catheterizations, previously placed stents were dilated, and a total of 6 additional telescoping stents were placed. The other patient underwent 3 reinterventions, with subsequent intervention at 9, 19, and 33 months from initial dilation of an existing VSD. During the additional 3 catheterizations, a total of 3 telescoping stents were placed, with 1 additional stent placed at each procedure. Hemodynamic findings and results of repeat intervention are shown in Figure 3. None of the previously placed stents were found to be fractured or embolized. Angiography demonstrated only mild degrees of in-stent stenosis, but recurrent obstruction occurred beyond

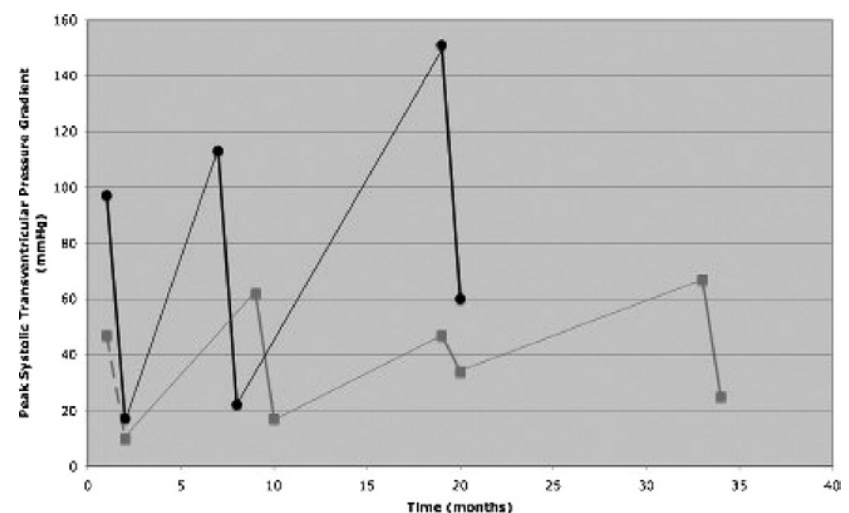

Figure 3. Results of repeated intervention. One patient (circles) initially underwent transcatheter ventricular septal defect (VSD) creation and stenting with repeat dilation and stenting at all subsequent procedures. The second patient (squares) underwent transcatheter enlargement of an existing VSD. The initial procedure involved only balloon dilation of the existing VSD without stenting (dashed lines). Subsequent interventions involved both repeat dilation and stenting (solid lines).

the proximal and distal ends of the stents, presumably caused by progressive ventricular hypertrophy. In nearly all cases, transventricular pressure gradients had returned to values exceeding preintervention gradients of the prior catheterization but responded each time to the addition of further stents beyond those previously placed (Figure 4).

\section{Procedural Details and Additional Interventions} Mean procedure time was 274 minutes (range, 62-407 minutes). Mean total fluoroscopy time was 117 minutes (range, 34-269 minutes). Mean contrast load was $8.3 \mathrm{~mL} / \mathrm{kg}$ (range, $5.2-11.8 \mathrm{~mL} / \mathrm{kg}$ ). Additional interventions were performed in 2 patients and included coarctation dilation, stenting of muscular subaortic stenosis, coil embolization of collateral vessels, and Fontan fenestration closure.

\section{Complications}

There was no procedural mortality, free-wall perforation, or pericardial effusion. Two patients experienced moderate-tosevere AVV regurgitation, with 1 patient requiring surgical repair. There were no cases of complete heart block requiring pacemaker placement. One patient had transient left bundle branch block, and 1 patient sustained several minutes of complete atrioventricular block, which was hemodynamically well tolerated and required no intervention. Nonsustained ventricular ectopy was common and required no specific therapy. Stent embolization occurred in 1 patient during an unrelated intervention, and the stent was safely repositioned. One patient incurred an air embolism related to balloon rupture, resulting in transient ST-segment changes. 


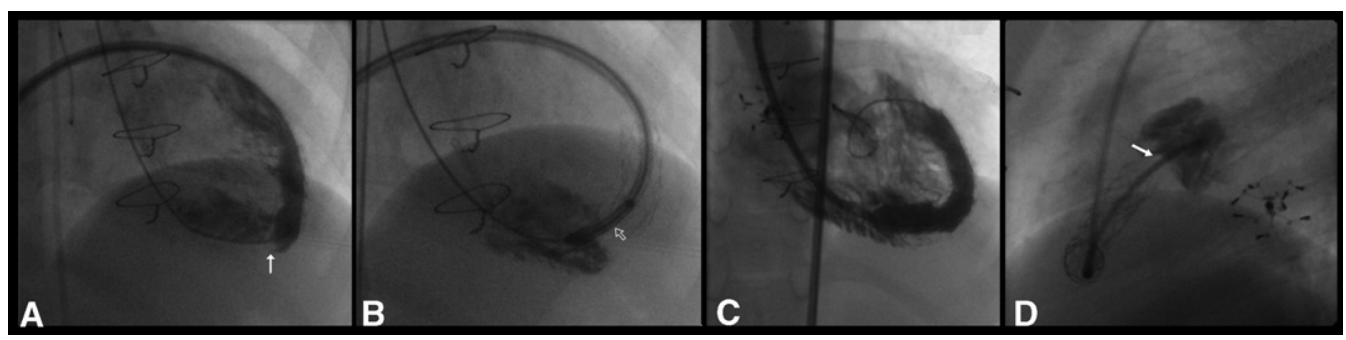

Figure 4. Mechanism of recurrent obstruction after ventricular septal defect (VSD) creation and stenting. After VSD creation and stenting, muscular obstruction occurred distal to the stent on the systemic right ventricular side (arrow, A). An additional stent is positioned (open arrow, B) and deployed (C) for relief of the obstruction. However, at repeat catheterization, muscular obstruction had occurred beyond the stent in the nonsystemic hypertensive ventricle (arrow, D).

\section{Follow-up}

Median follow-up from catheterization to most recent clinical evaluation or surgical intervention on the ventricular septum was 9 months (range, 14 days-114 months). All patients were alive, and no stent was fractured.

- Patient A, with a ventricular aneurysm and history of a cerebrovascular accident, underwent a total of 4 interventions. Nine years after the most recent intervention, the VSD stents were in good position, as determined by means of two-dimensional echocardiography, and the LV aneurysm had decreased in size. The estimated transseptal gradient, as determined by means of Doppler investigation, was 80 to $85 \mathrm{~mm} \mathrm{Hg}$, with continued severe dysfunction of the hypertensive ventricle.

- Patient B had a ventricular aneurysm and underwent a single procedure with dilation and stenting of an existing VSD. This patient had severe regurgitation of the nonsystemic AVV during the procedure and underwent surgical mitral valve repair 1 week later. At the time of the operation, there was a 10 to $15 \mathrm{~mm} \mathrm{Hg}$ Doppler gradient across the VSD stent. No further follow-up is available.

- Patient $\mathrm{C}$ underwent a single procedure with stenting of an existing VSD, as well as stenting of a muscular subaortic stenosis. Despite effective ventricular decompression, the patient had severe protein-losing enteropathy several months after his procedure, ultimately requiring cardiac transplantation. Neither the ventricular septum nor the subaortic area was interrogated before transplantation.

- Patient D underwent a single intervention consisting of VSD creation and dilation. This patient underwent takedown of obstructing mitral valve attachments during performance of a scheduled bidirectional Glenn operation 3 days after catheterization. At last follow-up 2 years after the operation, the patient had undergone the Fontan procedure, and the VSD was unrestrictive, as determined by means of Doppler echocardiography.

- Patient E had a ventricular aneurysm and underwent a single procedure with VSD creation and stenting. Thirty-three months of follow-up were available. At the time of the last clinical evaluation, the VSD stents were in good position, as determined by means of two-dimensional echocardiography. Adequate Doppler envelopes could not be obtained for estimation of residual VSD gradient. The LV aneurysm identified at catheterization was not seen by means of either preprocedural or postprocedural echocardiography.

- Patient F underwent VSD creation and stenting at the initial catheterization. Two subsequent interventions involved placement of 6 additional stents. At 22 months of follow-up, the VSD stents were in good position. The stented VSD remained patent, although severely obstructed, with a Doppler-estimated transseptal gradient of $200 \mathrm{~mm} \mathrm{Hg}$. Biventricular function remains preserved, and the dimensions of the hypertensive ventricle have remained stable, as determined by means of magnetic resonance imaging.

- Patient G's procedure was very recently performed, consisting of VSD creation and stenting, followed by Fontan fenestration closure. At follow-up evaluation 5 months later, the patient was clinically well. There remained no discernable gradient across the VSD stent, as determined by means of Doppler echocardiography.

- Patients H's procedure was the most recent procedure performed, consisting of VSD creation and stenting. Echocardiography during clinical follow-up 2 weeks later demonstrated a $21 \mathrm{~mm} \mathrm{Hg}$ Doppler-estimated gradient across the ventricular septum and minimal AVV regurgitation. 


\section{Discussion}

Most children with DORV are able to undergo biventricular repair. An uncommon subset requires palliation to a singleventricle physiology, most frequently those in association with complex abnormalities of the AVVs, noncommitted VSDs, and ventricular hypoplasia. ${ }^{1}$ Establishment of a univentricular physiology in these patients does not exclude the nonsystemic ventricle from physiologic importance, and accurate characterization of the VSD remains essential. Indeed, as early as 1952, Edwards and colleagues ${ }^{2}$ described the consequences of spontaneous LV outflow obstruction in a patient with DORV. Rao and Sissman ${ }^{3}$ subsequently articulated the more general principle of the "physiologically advantageous VSD" and identified morbidities associated with spontaneous closure. These and other early case reports form what remains a limited clinical experience with VSD closure in patients with DORV. ${ }^{4-9} \mathrm{We}$ have found that this complication is not unique to patients with DORV and can occur in any patient in whom the native ventricular outflow tract is either congenitally or iatrogenically absent, malpositioned, or severely obstructed, and the VSD constitutes the only means of egress from the nonsystemic ventricle. An unrestrictive atrial communication in these patients averts dramatic clinical presentation but does not obviate the need for intervention. Published case reports in this situation are limited but suggest poor outcomes in the absence of intercession or with incomplete decompression. ${ }^{8,10,11}$ On the basis of this limited information, physicians managing patients with isolated hypertensive ventricles have referred patients for surgical decompression, despite the general risks associated with surgical intervention and the specific risks inherent to this procedure, including those attendant reoperation in patients with Fontan physiology and hypertrophied ventricles. Surgical trauma to the atrioventricular node and accompanying heart block remain significant risks with VSD creation/enlargement and are related primarily to the location of the VSD and the extent of VSD enlargement necessary to achieve adequate ventricular decompression. Ideally, a VSD could be created in the mid to apical muscular septum, remote from conduction tissue. Transcatheter approaches allow unique visibility of, and access to, this part of the septum. Furthermore, when restriction across an existing VSD is secondary to AVV tissue, enlargement of this defect risks loss of valve function. In this setting creation of an additional muscular defect might pose less risk of disrupting the AVV than dividing attachments.

We used transcatheter techniques to create or enlarge VSDs in 8 patients with isolated and hypertensive ventricles. Despite efforts to minimize risk to the AVV during the procedure, 1 patient experienced AVV damage requiring surgical repair. Although it is unclear at exactly what point in the procedure the valve damage occurred, the surgical findings suggested that it was the catheter course and the combination of stiff wire, catheter, and sheath that likely tore the valve leaflet. The stented VSD was remote from the valve and was left unaltered at the time of the operation. Catheter course-related complications might be avoided by shaping equipment more specifically to the intended course or by maintaining a broad wire loop through the AVV and VSD during manipulations necessary for balloon dilation and stenting. In the future, a hybrid perventricular approach to creating midmuscular VSDs is likely to lessen the risk of AVV damage. Although this is a small series, none of the patients undergoing VSD creation/enlargement experienced more than transient procedural conduction disturbance.

Because of the rarity of the underlying anatomy and the poorly defined risks of the isolated hypertensive left ventricle, the clinical benefit is impossible to determine, particularly in this very small group of patients. Benefit might be limited to those patients with established associated morbidities. In our series 3 patients had associated ventricular aneurysms, and 2 of these patients had severely decreased function of the affected ventricle. To our knowledge, ventricular aneurysms have not been described previously in patients with this physiology, and as such, the pathogenesis of ventricular aneurysms in the present situation is not well delineated. The combined effects of chronic subendocardial ischemia and ventricular hypertension form an attractive hypothetical substrate for the development of ventricular aneurysms in this patient population, but this remains speculative. The prognosis of ventricular aneurysms in this situation is unclear. Review of the literature regarding congenital ventricular aneurysm reveals a very poor prognosis and no known incidence of spontaneous resolution. ${ }^{12-14}$ Inadequate follow-up precludes meaningful assessment of the affect of transcatheter ventricular decompression on aneurysms in 2 patients. In the 1 patient with adequate follow-up, the ventricular aneurysm had regressed in size.

Although we were able to successfully decompress isolated ventricles acutely, recurrent obstruction was common, and in 2 patients maintenance of an unrestrictive VSD required repeated interventions. The mechanism of reobstruction was similar in both cases and was related to continued ventricular hypertrophy and muscular obstruction beyond the ends of the stents. Significant neointimal ingrowth, myocardial prolapse, and stent fracture were not seen as mechanisms of reobstruction in our group. When reobstruction occurred, extension of the stented defects by means of further stent placement resulted in reduced obstruction. We hypothesize that avoidance of recurrent obstruction requires complete resolution of obstruction and ventricular hypertension and with it the incentive for progressive ventricular hypertrophy. Only in one of our most recent patients have we been able to completely equalize ventricular pressures after VSD creation. Follow-up in this 
patient remains limited, but at the most recent evaluation, there was no evidence of recurrent obstruction.

\section{References}

1. Russo P, Danielson GK, Puga FJ, McGoon DC, Humes R. Modified Fontan procedure for biventricular hearts with complex forms of double-outlet right ventricle. Circulation. 1988;78(suppl):III20-5.

2. Edwards JE, James JW, Du SJ. Congenital malformation of the heart; origin of transposed great vessels from the right ventricle associated with atresia of the left ventricular outlet, double orifice of the mitral valve, and single coronary artery. Lab Invest. 1952;1:197-207.

3. Rao PS, Sissman NJ. Spontaneous closure of physiologically advantageous ventricular septal defects. Circulation. 1971;43:83-90.

4. Serratto M, Arevalo F, Goldman EJ, Hastreiter A, Miller RA. Obstructive ventricular septal defect in double outlet right ventricle. Am J Cardiol. 1967;19:457-63.

5. Lavoie R, Sestier F, Gilbert G, Chameides L, Van Praagh R, Grondin $P$. Double outlet right ventricle with left ventricular outflow tract obstruction due to small ventricular septal defect. Am Heart J. 1971; 82:290-9.

6. Marin-Garcia J, Neches WH, Park SC, Lenox CC, Zuberbuhler JR, Bahnson HT. Double-outlet right ventricle with restrictive ventricular septal defect. J Thorac Cardiovasc Surg. 1978;76:853-8.
7. Cavalini JF, Aiello VD, Guedes de Souza P, Trevisan IV, Marcial MB, Ebaid M. Double outlet right ventricle with intact atrial septum and restrictive ventricular septal defect: an analysis of two cases. Pediatr Cardiol. 1998;19:490-4.

8. Marino B, Loperfido F, Sardi CS. Spontaneous closure of ventricular septal defect in a case of double outlet right ventricle. Br Heart J. 1983;49:608-11.

9. Mason DT, Morrow AG, Elkins RC, Friedman WF. Origin of both great vessels from the right ventricle associated with severe obstruction to left ventricular outflow. Am J Cardiol. 1969;24:118-24.

10. Matsuoka Y, Akimoto K, Sennari E, Hayakawa K. Double outlet right ventricle with severe left ventricular outflow tract obstruction due to small ventricular septal defect and anomalous adherence of the mitral valve to the ventricular septum. Jpn Circ J. 1987;51:1335-40.

11. Belli E, Serraf A, Lacour-Gayet F, Prodan S, Piot D, Losay J, et al. Biventricular repair for double-outlet right ventricle. Results and longterm follow-up. Circulation. 1998;98(suppl):II360-7.

12. Marijon E, Ou P, Fermont L, Concordet S, Le Bidois J, Sidi D, et al. Diagnosis and outcome in congenital ventricular diverticulum and aneurysm. J Thorac Cardiovasc Surg. 2006;131:433-7.

13. Folger GM Jr, Stanton PE. Annular subvalvular left ventricular aneurysm in a North American infant. Am Heart J. 1971;81:392-7.

14. Abrahams DG, Barton CJ, Cockshott WP, Edington GM, Weaver EJ. Annular subvalvular left ventricular aneurysms. $Q J$ Med. 1962;31: $345-60$. 